\title{
Campañas electorales y Twitter. La difusión de contenidos mediáticos en el entorno digital
}

\section{Electoral campaigns and Twitter. The dissemination of media content in the digital environment \\ Campanhas eleitorais e Twitter. A disseminação do conteúdo midiático no ambiente digital}

Silvia Marcos-García, Universitat Jaume I de Castelló, Castellón de la Plana, España (smarcos@uji.es)

Laura Alonso-Muñoz, Universitat Jaume I de Castelló, Castellón de la Plana, España (lalonso@uji.es)

Amparo López-Meri, Universitat Jaume I de Castelló, Castellón de la Plana, España (meri@uji.es)

RESUMEN | Durante las últimas décadas, las redes sociales han transformado de forma relevante el campo de la comunicación política. Partidos y políticos han incorporado estas plataformas como una herramienta habitual en su estrategia comunicativa. A su vez, los medios de comunicación convencionales siguen manteniendo un rol central en la esfera política y social. En este contexto marcado por la convivencia entre medios tradicionales y medios sociales, los actores políticos aprovechan las potencialidades de ambos espacios comunicativos para ampliar la difusión de su mensaje. Esta investigación examina la relación entre medios convencionales y Twitter durante la campaña electoral de 2016 en España. La metodología combina el análisis de contenido cuantitativo y el análisis métrico sobre una muestra de 8199 mensajes publicados por los cuatro partidos más votados: PP, PSOE, Podemos y Ciudadanos. Los resultados confirman que los partidos aprovechan Twitter para amplificar el impacto de sus intervenciones en medios convencionales, siendo especialmente significativo el debate a cuatro emitido por diferentes cadenas televisivas. Este dato refuerza la idea de que viejos y nuevos medios conviven y se complementan en un entorno comunicativo híbrido.

PALABRAS CLAVE: comunicación política; campaña electoral; medios sociales; Twitter; televisión; hibridación. 
ABSTRACT | During the last decades, social media have significantly transformed the field of political communication. Political parties and politicians have incorporated these platforms as a regular tool in their communication strategy. In turn, the mainstream media continues to play a key role in the political and social sphere. In this context marked by the coexistence between traditional media and social media, political actors take advantage of the potential of both communication spaces to expand the dissemination of their message. This research examines the relationship between mass media and Twitter during the 2016 election campaign in Spain. The methodology combines the quantitative content analysis and the metric analysis on a sample of 8199 messages published by the four most voted political parties: PP, PSOE, Podemos and Ciudadanos. The results confirm that parties take advantage of Twitter to amplify the impact of their interventions in mass media, being especially significant the debate broadcast on television. This data reinforces theidea that old and new media coexist and complement each other in a hybrid communicative environment.

KEYWORDS: political communication; election campaign; social media; Twitter; television; hybridization.

RESUMO|Nas últimas décadas, as redes sociais transformaram significativamenteo campo da comunicação política. Partidos e políticos incorporaram essas plataformas como uma ferramenta regular na sua estratégia de comunicação. Por sua vez, a grande mídia continua a desempenhar um papel central na esfera política e social. Nesse contexto marcado pela coexistência entre mídias tradicionais e mídias sociais, os atores políticos aproveitam o potencial dos dois espaços de comunicação para expandir a disseminação da sua mensagem. Esta pesquisa examina a relação entre a mídia convencional e o Twitter na campanha eleitoral de 2016 na Espanha. A metodologia combina a análise quantitativa de conteúdo e a análise métrica em uma amostra de 8.199 mensagens publicadas pelos quatro partidos políticos mais votados: PP, PSOE, Podemos e Ciudadanos. Os resultados confirmam que os partidos aproveitam o Twitter para ampliar o impacto de suas intervenções na mídia convencional, sendo especialmente significativo o debate desses partidos veiculado na televisão. Esses dados reforçam a ideia de que os meios tradicionais e novos coexistem e se complementam em um ambiente comunicativo híbrido.

PALAVRAS-CHAVE: comunicação política; campanha eleitoral; redes sociais; Twitter; televisão; hibridização. 


\section{INTRODUCCIÓN}

El empleo de las redes sociales, especialmente de Twitter, es actualmente un recurso habitual en el campo de la comunicación política. Las potencialidades de la red de microblogging, donde prima la facilidad de participación y de interacción entre usuarios, hacen de esta plataforma una herramienta preferente tanto para políticos, como para periodistas y ciudadanos (Parmelee \& Bichard, 2011; Kruikemeier, 2014). En este sentido, Twitter se ha convertido en un nuevo medio para la deliberación de las principales cuestiones políticas y procesos democráticos que afectan a la sociedad (Chadwick, 2011).

Las campañas electorales que se han celebrado a lo largo de esta última década han demostrado el rol destacado que ha jugado Twitter en las estrategias comunicativas de los actores políticos. Tanto partidos como políticos han empleado este medio social como un canal para difundir información sobre actos de campaña, compartir declaraciones políticas, movilizar el voto y conseguir la participación de su electorado (Jackson \& Lilleker, 2011; Parmelee \& Bichard, 2011; Gainous \& Wagner, 2014; López-Meri, Marcos-García, \& Casero-Ripollés, 2017), así como para dialogar con otros usuarios desde una óptica personal, incluso humorística, dando a conocer emociones o aspectos de su vida privada (Vergeer, Hermans, \& Sams, 2013).

Los partidos también han aprovechado Twitter como un altavoz desde el que promocionar y viralizar sus intervenciones en programas de televisión, entrevistas en diarios y cualquier otro contenido sobre ellos producido por los medios de comunicación convencionales. De este modo, emplean esta red social como un espacio híbrido en el que usan indistintamente las lógicas de los viejos y los nuevos medios de forma simultánea (Casero-Ripollés, Feenstra, \& Tormey, 2016; Chadwick, 2017).

El objetivo general de esta investigación es evaluar la relación entre medios sociales y medios convencionales durante una campaña electoral a partir del estudio de los picos o jornadas de máxima actividad en Twitter de los cuatro partidos más votados en las elecciones generales de 2016 en España. Se analizan los perfiles de dos partidos clásicos, Partido Popular (PP) y Partido Socialista Obrero Español (PSOE), y de dos formaciones emergentes, Podemos y Ciudadanos. Con ello, se pretende identificar qué tipo de acontecimientos provocan una mayor producción de tuits, para conocer si existe una conexión directa entre los eventos mediados y el nivel de publicaciones de los partidos.

Hasta el momento, las investigaciones basadas en el estudio de la hibridación en la comunicación política se han centrado en el uso de Twitter por parte de los ciudadanos. Específicamente, han focalizado su atención en el grado de actividad y en el tipo y función de los mensajes lanzados por estos usuarios en relación a un 
evento político mediado y retransmitido en directo como, por ejemplo, los debates electorales (Shamma, Kennedy, \& Churchill, 2009; Wohn \& Na, 2011; Mascaro \& Goggins, 2012; Freelon \& Karpf, 2015; Marcos-García, Alonso-Muñoz, \& CaseroRipollés, 2016). Sin embargo, todavía son pocos los estudios que examinan cómo los actores políticos emplean Twitter para enfatizar y viralizar su participación en tertulias televisivas, programas de radio y otros contenidos centrados en su figura y producidos por los medios de comunicación convencionales. Por ello, esta investigación aporta una visión novedosa que contribuye a ampliar el conocimiento en el campo de la comunicación y el empleo de las redes sociales por los actores políticos.

\section{LA COMUNICACIÓN POLÍTICA Y LAS CAMPAÑAS ELECTORALES EN TWITTER}

La incorporación de Twitter como herramienta comunicativa es un recurso habitualen las estrategias de partidos y políticos, especialmente durante los periodos de campaña electoral (Criado, Martínez-Fuentes, \& Silván, 2013; Suau-Gomila \& Pont-Sorribes, 2019). Junto a otras redes sociales como Facebook o YouTube, el uso de la red de microblogging en la comunicación política ha supuesto una completa transformación en los procesos de producción y distribución de la información, de forma que se han establecido nuevos paradigmas en el actual escenario político (Doménech, 2018). En este sentido, los medios sociales ya no solo han ampliado los espacios donde los actores políticos y mediáticos pueden lanzar de forma eficiente su mensaje a un público mayor, sino que han multiplicado la tipología de actores que interactúan y negocian en el campo de la comunicación política (Casero-Ripollés, 2018). Estas plataformas ofrecen a los ciudadanos y ciudadanas la oportunidad de informarse, debatir y participar libremente (Jenkins, 2006; Ceccarini, 2015). Tal y como indican autores como Rúas y Casero-Ripollés (2018), la llegada de las redes sociales ha permitido a la ciudadanía contar con unos mecanismos para producir y difundir sus propios contenidos y poder participar, consecuentemente, en la conversación política. Este potencial introduce un panorama comunicativo mucho más competitivo que el existente en décadas anteriores que, además, se caracteriza por la aparición de nuevas formas de participación en los procesos democráticos (Chadwick, 2011).

En este contexto, los actores políticos han visto la necesidad de adaptar su estrategia comunicativa al entorno digital para maximizar su capacidad para acercarse al electorado y valorar sus opiniones y comentarios de forma más directa (Stromer-Galley, 2014; Lilleker, Tenscher, \& Štětka, 2015). Esta potencialidad se explota sobre todo en Twitter, que se ha convertido actualmente en una de las plataformas sociales de referencia en la política virtual (Parmelee \& Bichard, 2011; 
Kruikemeier, 2014). Los medios sociales no solo han ayudado a partidos y políticos a gestionar su comunicación, sino también su imagen pública, ampliando así la gama de instrumentos que pueden explotar para su autopromoción (Rega, 2016). Por este motivo, autores como Piscitelli afirman que Twitter es "uno de los mecanismos de comunicación más poderosos de la historia” (2011, p. 15).

\section{CÓMO APROVECHAN LAS POTENCIALIDADES DE TWITTER LOS ACTORES POLÍTICOS}

La multitud de características que ofrece la red de microblogging la hacen una valiosa herramienta para los actores políticos. Entre otras posibilidades, permite lanzary recibirinformación a tiempo real (Jewitt, 2009), dirigir un mensaje a grandes grupos de usuarios (Kim, 2011), plantear una comunicación de naturaleza pública y sin restricciones (Rossi \& Magnani, 2012) y combinar métodos heterogéneos de conversación (Papacharissi \& De Fatima Oliveira, 2012; Murthy, 2013).

A partir del uso de Twitter, los partidos y políticos, así como otros usuarios, pueden enviar mensajes fácilmente a sus propios seguidores (retuit), vincularlos a otros usuarios a partir de las menciones (@), enlazar a contenidos externos (links) o participar en una conversación o debate sobre un determinado tema a partir del empleo de uno o varios hashtags relacionados (\#).

Si bien estas son algunas de las particularidades generales de Twitter, la literatura previa señala que las características inherentes de esta red social posibilitan a partidos y políticos otros usos específicos de la comunicación política. Así, una de las principales funciones de Twitter es ofrecer una vía que permite a los representantes e instituciones políticas comunicarse fácil, directa y masivamente con otros usuarios, principalmente, con su electorado (Hosch-Dayican, 2014; Rega, 2016). Una conversación que, además, se produce sin la necesidad de pasar por el filtro mediático que tradicionalmente dominaba la relación entre políticos y ciudadanos (Casero-Ripollés, 2008).

Sin embargo, pese a que cada vez son más los políticos y partidos que emplean Twitter como herramienta de comunicación, todavía son pocos los que la utilizan como un medio para relacionarse y dialogar con la ciudadanía (Alonso \& Adell, 2011; Alonso-Muñoz, Miquel-Segarra, \& Casero-Ripollés, 2016; Alonso-Muñoz, Marcos-García, \& Casero-Ripollés, 2016).

Otra función relevante que los actores políticos otorgan a Twitter es la promoción (López-Meri, Marcos-García, \& Casero-Ripollés, 2017). En este sentido, emplean esta plataforma como un canal para informar de sus actividades de campaña, ofrecer declaraciones políticas, compartir enlaces a sus propias webs y evaluar su desempeño en los eventos en los que participan (Jackson \& Lilleker, 2011; Parmelee 
\& Bichard, 2012; Gainous \& Wagner, 2014). Twitter se ha convertido, incluso, en un reclamo para incentivar aportaciones económicas y movilizar al voluntariado en las campañas electorales (Norquay, 2008; Rodríguez \& Ureña, 2011). En definitiva, los políticos utilizan esta red social como un tablón de anuncios a través del cual transmiten los datos que les interesa hacer llegar directamente al electorado (Alonso \& Adell, 2011).

Las formaciones, y sobre todo sus líderes, emplean principalmente Twitter como un mecanismo para resaltar su imagen pública y humanizarse ante el resto de usuarios de esta red social (Jackson \& Lilleker, 2011; Holtz-Bacha, 2013; Bentivegna, 2015; Karlsen \& Enjolras, 2016). Así, a las propuestas programáticas y atributos profesionales, se suman otros contenidos basados en aspectos íntimos, vida privada o aficiones que estrechan y personalizan la relación entre los actores políticos y su electorado (Holtz-Bacha, 2013; Kruikemeier, 2014; Marcos-García \& Alonso-Muñoz, 2019).

Paralelamente, el uso que la clase política hace de Twitter concede una gran importancia a los medios de comunicación convencionales. Muchos actores políticos emplean esta plataforma para provocar titulares y ganar presencia en los diarios y en los programas de radio y televisión (Strömbäck, 2008; Broersma \& Graham, 2013), de forma que Twitter funciona como un canal de transmisión de contenidos mediáticos (Casero-Ripollés et al., 2016). En otras palabras, partidos y políticos utilizan esta red social para compartir con el resto de usuarios sus colaboraciones o apariciones en medios, con el objetivo de viralizar las entrevistas y noticias en las que son protagonistas, y que previamente han difundido periódicos, emisoras de radio o canales de televisión (Alonso-Muñoz \& Casero-Ripollés, 2018).

\section{TWITTER COMO ESPACIO HÍBRIDO EN LA COMUNICACIÓN POLÍTICA}

Desde la llegada de Internet y de las redes sociales, se han alzado numerosas conjeturas acerca de una posible desaparición de los medios de comunicación tradicionales, especialmente de la prensa escrita. Sin embargo, muchos autores afirman que los medios convencionales todavía desempeñan un papel crucial en la comunicación política (Chadwick, 2017). Recientes investigaciones señalan que medios sociales como Twitter son plataformas que resultan de especial relevancia en las rutinas profesionales de los medios y de las figuras políticas (Trilling, 2015). Como sucede con políticos y partidos, los mass media y sus profesionales siguen desempeñando un rol importante en la comunicación política y el entorno digital (Cha, Haddadi, Benevenuto, \& Gummadi, 2010; Casero-Ripollés, 2020). 
En este sentido, la red social de microblogging funciona como un espacio híbrido donde coexisten en equilibrio las lógicas de transmisión y recepción de los medios convencionales y las nuevas lógicas y potencialidades de circulación y negociación que ofrece el contexto digital (Chadwick, 2017; Graham, Broersma, Hazelhoff, \& van't Haar, 2013). En otras palabras, Twitter plantea un proceso de integración en el que nuevos y viejos medios conviven en un mismo hábitat, se nutren de sus contenidos propios y cooperan e interaccionan de forma simultánea y dinámica (Chadwick, 2011; Casero-Ripollés et al., 2016). Esta relación supone, para autores como Marchetti y Ceccobelli (2016), un impacto positivo en la arena política.

Respecto de los medios de comunicación, cada vez son más los que incorporan secciones y ofrecen numerosos contenidos basados en las publicaciones o declaraciones de un político en Twitter (Rodríguez \& Ureña, 2011; Marchetti \& Ceccobelli, 2016). Asimismo, los periodistas han pasado a considerar esta red social como una fuente relevante para encontrar información y como una vía para la curación de contenidos especializados (López-Meri \& Casero Ripollés, 2017).

En cuanto a los actores políticos, no han abandonado ni sustituido las tácticas y herramientas tradicionales de su estrategia comunicativa, sino que las enriquecen con las nuevas dinámicas y posibilidades que ofrecen los medios sociales (López-Meri et al., 2017). De esta manera, aprovechan la facilidad de interacción característica de Twitter para dialogar con los medios de comunicación y sus profesionales (Jungherr, 2014). Además, los políticos explotan recursos propios de esta red social, como los hashtags y las menciones a periodistas y a espacios mediáticos en los que intervienen (Alonso-Muñoz et al., 2016).

Esta dinámica, tal y como reflejan investigaciones previas, obtiene una gran respuesta por parte de los usuarios. Los picos o días de máxima actividad en Twitter suelen coincidir con la difusión de contenidos políticos en los medios de comunicación convencionales (Hanna, Wells, Maurer, Friedland, Shah, \& Matthes, 2013; Larsson, 2016), especialmente en televisión. Twitter ha pasado a funcionar como un canal donde políticos, ciudadanos y periodistas pueden interactuar y compartir sus interpretaciones, opiniones y comentarios durante las emisiones televisivas (Marchetti \& Ceccobelli, 2016). Autores como Chadwick (2011) o Iannelli (2016) afirman, incluso, que los políticos aprovechan la retransmisión de eventos políticos televisados para formar comunidades de usuarios mediante el uso de hashtags, trending topics o palabras clave relacionadas.

Esta tendencia gana importancia cuando se emiten los debates electorales, que registran el mayor número de retuits y comentarios de los usuarios (Anstead \& O’Loughlin, 2011). En este sentido, Twitter permite que las audiencias ya no solo 
consuman el contenido político a través de sus televisores, sino que participen y respondan al debate, entrando en la esfera pública desde sus propias casas (Pedersen, Baxter, Burnett, Goker, Corney, \& Martin, 2020). Twitter se ha convertido en un destacado backchannel para los debates políticos, de forma que las reacciones de la audiencia frente a las intervenciones de los actores políticos se hacen mucho más visibles en un contexto en línea (Kalsnes, Krumsvik, \& Storsul, 2014). Los actores políticos, por tu parte, aprovechan la viralidad de las redes sociales como un valor añadido, pues pueden difundir su mensaje por diferentes canales, llegando así a una mayor audiencia (Pérez-Curiel \& García-Gordillo, 2020). Por tanto, se observa cómo Twitter plantea un nuevo entorno comunicativo híbrido, en el que tanto los contenidos emitidos por los medios convencionales, especialmente la televisión, como la participación de los usuarios en el contexto digital, están en una continua y constante retroalimentación.

\section{METODOLOGÍA}

Este trabajo se desarrolla en dos fases. La primera se destina a identificar los picos de actividad de cada partido en Twitter. Los picos de actividad hacen referencia a las jornadas que superan la media diaria de tuits publicados por cada formación. Para ello, se realiza un análisis métrico de los perfiles de los cuatro partidos seleccionados (Partido Popular, Partido Socialista, Podemos y Ciudadanos), correspondientes a las cuatro formaciones que obtuvieron un mayor número de votos en las elecciones generales de 2016 en España. La muestra incluye 8199 unidades de análisis, que comprenden todos aquellos mensajes lanzados por los perfiles analizados, así como las respuestas, pero no los retuits, dado que estos únicamente sirven para redifundir los contenidos publicados por terceros autores (Larsson, 2017). Se trata de la suma de tuits difundidos por el Partido Popular (PP), Partido Socialista (PSOE), Podemos y Ciudadanos durante 18 días, comprendidos entre el 10 de junio (inicio de la campaña electoral) y el 27 de junio (día posterior a la votación). La muestra se ha capturado con la aplicación web Twitonomy.

La segunda fase de esta investigación se centra en los picos o jornadas de máxima actividad de cada partido. Específicamente, se realiza un análisis de contenido cuantitativo de los tuits publicados en estas fechas con el objetivo de evaluar, entre otros factores, si los picos de actividad en Twitter coinciden con la celebración de eventos mediados por medios convencionales. En cada partido, según el caso, se han detectado de 8 a 11 jornadas que superan la media diaria de tuits. La muestra correspondiente a estos picos de actividad asciende a 5728 publicaciones (tabla 1). 


\begin{tabular}{cccc} 
Partido & Total tuits & En los picos & $\mathbf{N}^{\circ}$ picos \\
\hline PP & 995 & 789 & 11 días \\
\hline PSOE & 3113 & 2268 & 9 días \\
\hline Podemos & 2511 & 1617 & 8 días \\
\hline Ciudadanos & 1580 & 1054 & 9 días \\
\hline Total & 8199 & 5728 & \\
\hline
\end{tabular}

Tabla 1. Desglose de la muestra de tuits de la campaña electoral de 2016

Fuente: Elaboración propia.

El análisis de contenido cuantitativo persigue examinar el rol que juegan en Twitter las intervenciones de los líderes y de otros miembros de cada formación en los medios de comunicación convencionales, respecto de otros mensajes centrados en los actos de campaña o en el programa electoral. Para ello, se creó un libro de códigos en el que se incluyeron cinco variables: 1) el tipo de contenido compartido; 2) la presencia en medios de comunicación; 3) el tipo de programa en el que se participaba; 4) los hashtags empleados en relación con los programas, y 5) las menciones empleadas relacionadas con los medios de comunicación y los programas en los que aparecían. De este modo, se trata de responder a tres preguntas de investigación:

PI1. ¿Coinciden los picos de actividad de los partidos en Twitter con la celebración de eventos mediados por medios convencionales?

PI2. ¿Qué tipo de medio tiene mayor protagonismo cuando se producen picos de actividad en los perfiles de los partidos en Twitter?

PI3. ¿Existen diferencias entre partidos clásicos y partidos emergentes en relación con la convivencia entre viejos $y$ nuevos medios?

Respecto de estas cuestiones, se establecen las siguientes hipótesis:

H1. Los picos de actividad de los partidos en Twitter durante la campaña electoral suelen coincidir con eventos mediados por medios convencionales.

H2. La televisión tiene más protagonismo que la radio y la prensa cuando se producen picos de actividad en los perfiles de los partidos en Twitter.

H3. Los partidos emergentes apuestan por la televisión, mientras que los partidos clásicos reparten más equitativamente el espacio, sin olvidar a la radio y a la prensa. 
El análisis se ha realizado mediante el uso del paquete estadístico SPSS (v.23). La muestra fue analizada manualmente por tres miembros del equipo de investigación. Para calcular el grado de fiabilidad entre los codificadores, se analizaron 600 mensajes relativos del total de la muestra que hace referencia a los picos de actividad ( $\mathrm{N}=5728$ ), lo que implica aproximadamente $10 \%$ de la muestra, alcanzado un nivel de fiabilidad de 0,95 según el coeficiente Pi de Scott.

\section{RESULTADOS}

El primer dato destacado es la desigualdad entre partidos en cuanto a la frecuencia de publicación en Twitter. En la campaña electoral de 2016, PSOE y Podemos son las formaciones más activas, con 3113 y 2511 tuits respectivamente. Ciudadanos y PP se sitúan en un nivel inferior, con 1580 y 995 unidades respectivamente. Por lo tanto, la media diaria de publicaciones durante los 18 días analizados es mayor en los perfiles de PSOE (172 tuits) y Podemos (139 tuits), que en las cuentas de Ciudadanos (87 tuits) y PP (55 tuits).

En este sentido, se han detectado, según el caso, entre ocho y once picos de actividad o días en los que cada partido supera su media diaria de actualizaciones (figura 1). El PP es el más constante. Publica menos y, por eso, su media diaria es más reducida. Por este motivo, el partido liderado por Mariano Rajoy presenta once picos de mayor actividad, una cifra destacada si se considera que el periodo temporal analizado es de 18 días. Por su parte, PSOE y Ciudadanos superan su media diaria en nueve ocasiones, y Podemos lo hace en ocho jornadas. No obstante, solo cinco de los picos de actividad registrados son comunes a las cuatro formaciones: los días 13, 15, 16, 23 y 24 de junio (figura 1).

Los picos más abruptos se ocasionan, por un lado, el 13 de junio, coincidiendo con el debate a cuatro producido por la Academia de Televisión, en colaboración con RTVE, Atresmedia y Mediaset, y emitido por 17 canales, y, por otro lado, el 24 de junio, con el cierre de la campaña electoral. Ese último día de campaña, PSOE y Podemos superan los 300 tuits, mientras que PP y Ciudadanos rondan el centenar de mensajes. En cuanto al resto de días, 15, 16 y 23 de junio, el número de actualizaciones se aproxima a la media. Por este motivo, en estas fechas, el gráfico refleja menos oscilaciones. 


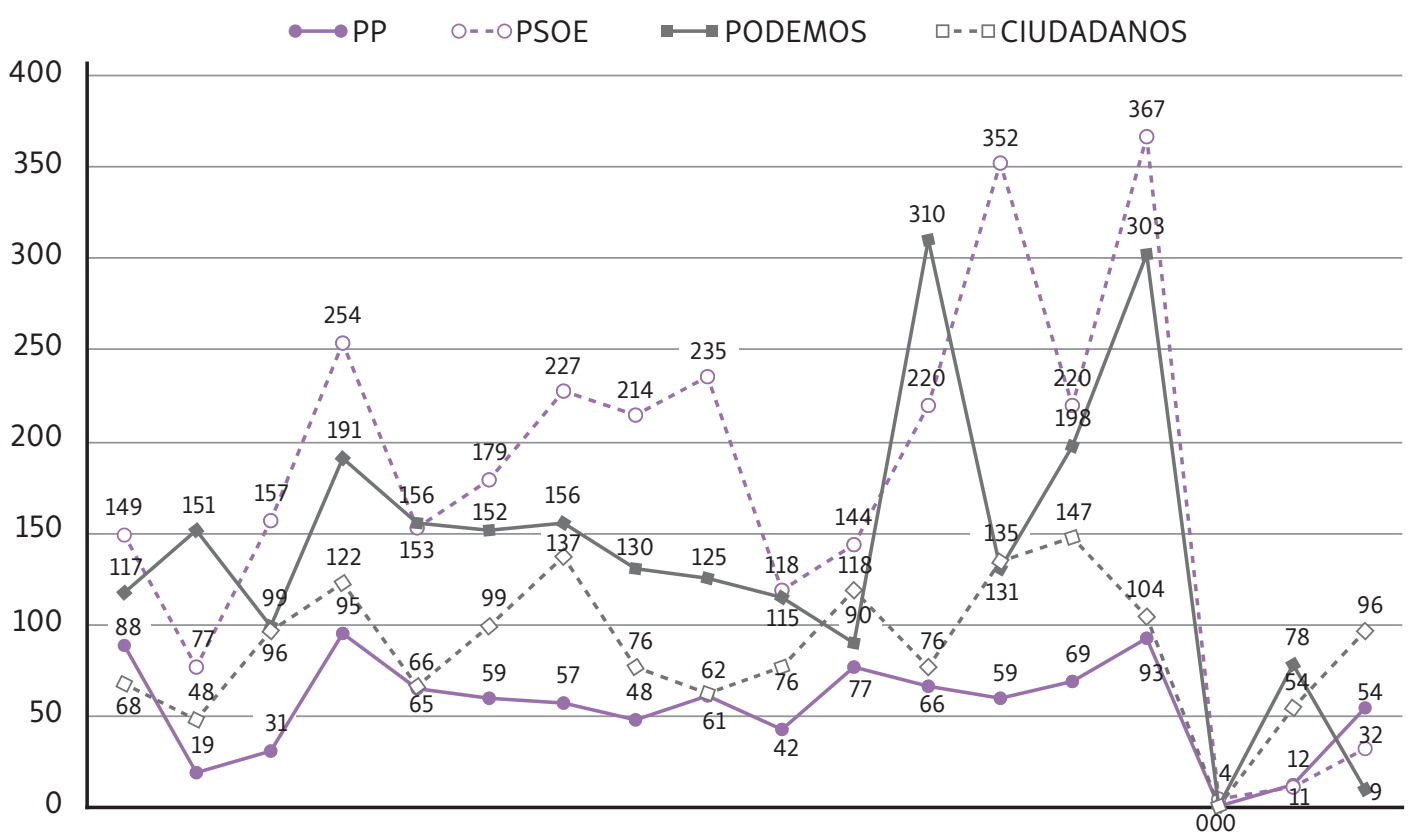

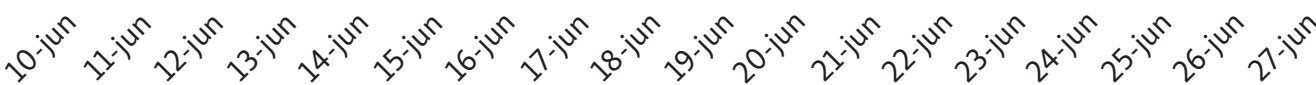

Figura 1. Distribución diaria de los tuits publicados en la campaña electoral de 2016

Fuente: Elaboración propia.

\section{RELACIÓN ENTRE PICOS DE ACTIVIDAD EN TWITTER Y CONTENIDOS MEDIÁTICOS}

Respecto de la confluencia de picos de actividad y eventos difundidos en medios de comunicación convencionales, existe mayor coincidencia en los casos de PP y Ciudadanos (figura 2). En ambos perfiles, las intervenciones en medios de comunicación, tanto del candidato como de otros miembros del partido, protagonizan cerca de la mitad de los mensajes (entre $46 \%$ y $48 \%$ ).

En cambio, las cuentas del PSOE y Podemos dedican más espacio a retransmitir sus mítines y actos de campaña (entre 63\% y $64 \%$ de los tuits) que a difundir sus apariciones en medios (entre $26 \%$ y $28 \%$ ). El programa electoral pasa inadvertido (entre $4 \%$ y $10 \%$ de los tuits), excepto en el caso de PP (31\%).

Si se analiza cada pico de actividad por separado, se aprecian tendencias y detalles específicos de cada formación. Se constata que el debate a cuatro entre los candidatos colapsó el timeline de los partidos el 13 de junio. Este es el día que más atención prestaron en Twitter a las intervenciones en medios (93\% de los tuits de Podemos; $82 \%$ de Ciudadanos; $76 \%$ de PP y $61 \%$ de PSOE). Además, todos los partidos coinciden al incluir en sus tuits el hashtag específico del debate, \#Debate13J, cuando destacan las declaraciones de sus respectivos líderes. 


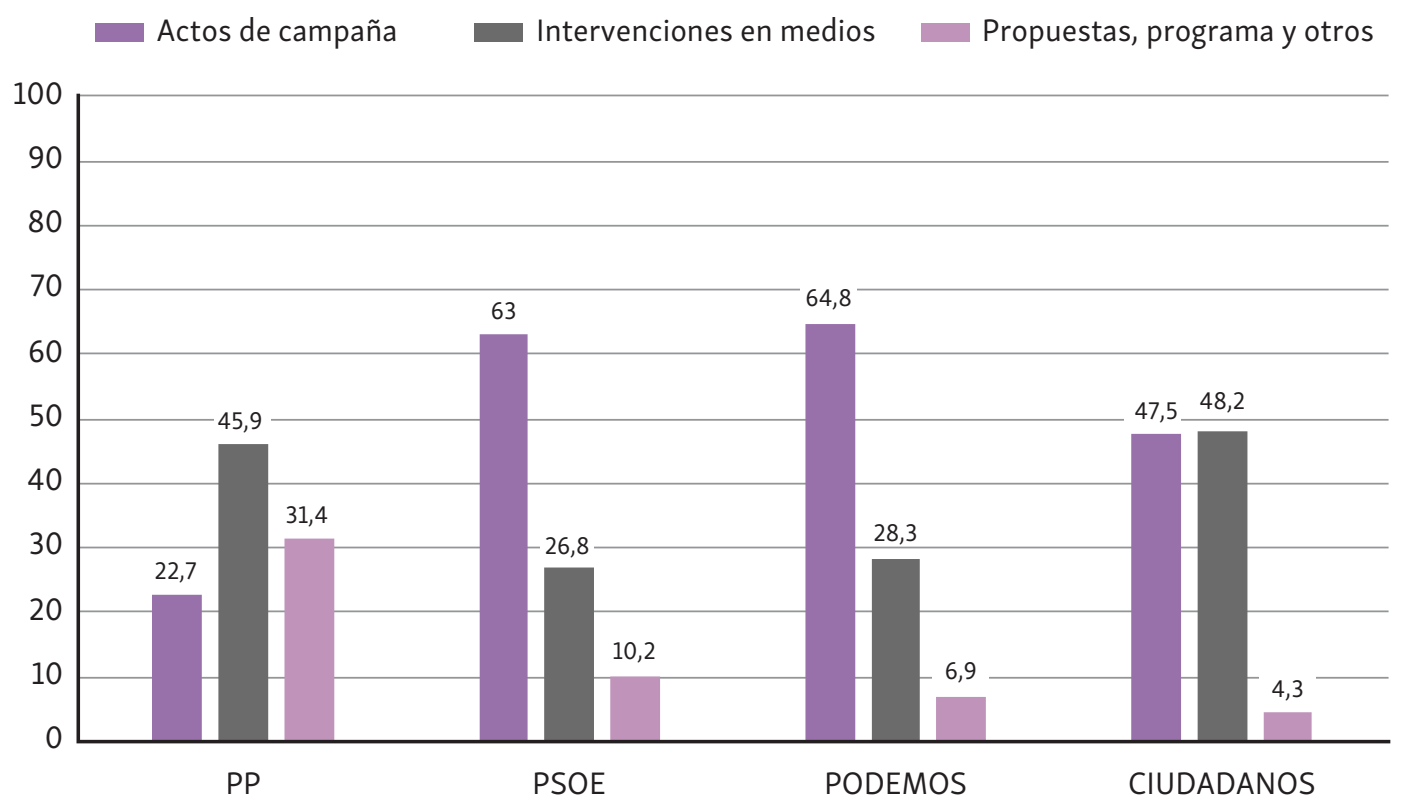

Figura 2. Contenidos difundidos durante los días de más actividad (\%)

Fuente: Elaboración propia.

Por otro lado, PSOE y Podemos, pese a que dedican un número importante de mensajes a promocionar contenidos elaborados por los medios convencionales (608 y 458 tuits respectivamente), estos no tienen demasiado peso en relación al número total de publicaciones. Al margen del día del debate, solo cuentan con otra jornada que alcanza cierta notoriedad por su vinculación a contenidos mediáticos. En el caso de Podemos, se trata del 14 de junio, cuando dedica $48 \%$ de los tuits a promocionar sus intervenciones en seis programas de radio y televisión. En cuanto al PSOE, el día señalado es el 15 de junio, e invierte $40 \%$ de los mensajes en cinco espacios mediáticos.

Por su parte, PP y Ciudadanos se centran en contenidos mediáticos con mayor frecuencia que PSOE y Podemos. Específicamente, las alusiones a medios convencionales superan la mitad de los tuits del PP en cinco jornadas (días 10, 13, 20, 21 y 22 de junio). Lo mismo sucede en la cuenta de Ciudadanos en cuatro ocasiones (días 13, 16, 20 y 27 de junio).

Por ejemplo, PP promociona sus apariciones hasta en cinco medios distintos en una misma jornada (10 de junio). Asimismo, Ciudadanos, mucho más activo en este ámbito, llega a informar sobre trece intervenciones en medios en un único día (27 de junio).

Además, en las cuentas de PP y Ciudadanos destaca el 20 de junio, fecha en la que Televisión Española (TVE) emitió un debate a siete, al que se sumaron Esquerra Republicana de Cataluña (ERC), Convergència Democràtica de Catalunya (CDC) y 
Partido Nacionalista Vasco (PNV). En este debate se prescindió de los líderes de los principales partidos, enfrentando a los portavoces de estas formaciones, como Pablo Casado (PP) o Juan Carlos Girauta (Ciudadanos).

En conjunto, hasta en once días de la campaña electoral alguno de los partidos dedica al menos $40 \%$ de su actividad en Twitter a compartir sus apariciones en medios convencionales. En siete jornadas, el porcentaje aumenta a 50\%. En cinco ocasiones, incluso se supera la barrera del 60\%. Estos datos refuerzan la idea de que los picos de actividad en esta red social, con frecuencia, están vinculados a la celebración de eventos mediados o a la promoción de contenidos previamente difundidos por los medios tradicionales.

\section{ANÁLISIS DE LA PRESENCIA DE MEDIOS EN LAS CUENTAS DE LOS PARTIDOS EN TWITTER}

La televisión es el medio con mayor presencia en Twitter durante la campaña electoral. El 33,8\% de los tuits publicados por los partidos durante los días de mayor actividad se refiere a medios de comunicación (1936 de 5728 unidades). En $69,3 \%$ de las ocasiones se alude a la televisión, en $22,7 \%$ a la radio y en $8 \%$ a la prensa. Si se analiza individualmente el perfil de cada partido, se aprecia que esta tendencia se repite en todos los casos (figura 3). Así, las entrevistas y declaraciones en programas televisivos están presentes en más de $60 \%$ de los contenidos mediáticos tuiteados por las cuatro fuerzas políticas.

PP y Podemos registran los porcentajes más altos, por encima de 70\%, rozando el $80 \%$ en el caso de Podemos. En parte, se debe a la importancia que adquieren los dos debates televisados, el 13 de junio con los cuatro candidatos y el 20 de junio con representantes de siete partidos. No obstante, los partidos también promocionan sus apariciones en las tertulias de los principales magacines matinales, como Espejo Público (Antena 3), El programa de Ana Rosa (Tele 5) y Las Mañanas de Cuatro, en programas de humor y entretenimiento, como El Hormiguero (Antena 3), o en espacios dedicados a la investigación periodística y el análisis, como El Objetivo (La Sexta). También se aprecia que prefieren citar programas específicos antes que mencionar los perfiles corporativos genéricos de las cadenas de televisión.

La presencia de contenidos radiofónicos es menor, ronda 20\%, excepto en el caso de Ciudadanos que alcanza un 30\%. Cadena SER y Onda Cero son dos de las emisoras más citadas, especialmente para anunciar la hora a la que sus seguidores podrán escuchar en directo al candidato o a otros portavoces del partido. Las alusiones a la prensa impresa o digital son menos frecuentes. En este caso, solo superan $10 \%$ en el perfil del PSOE. 


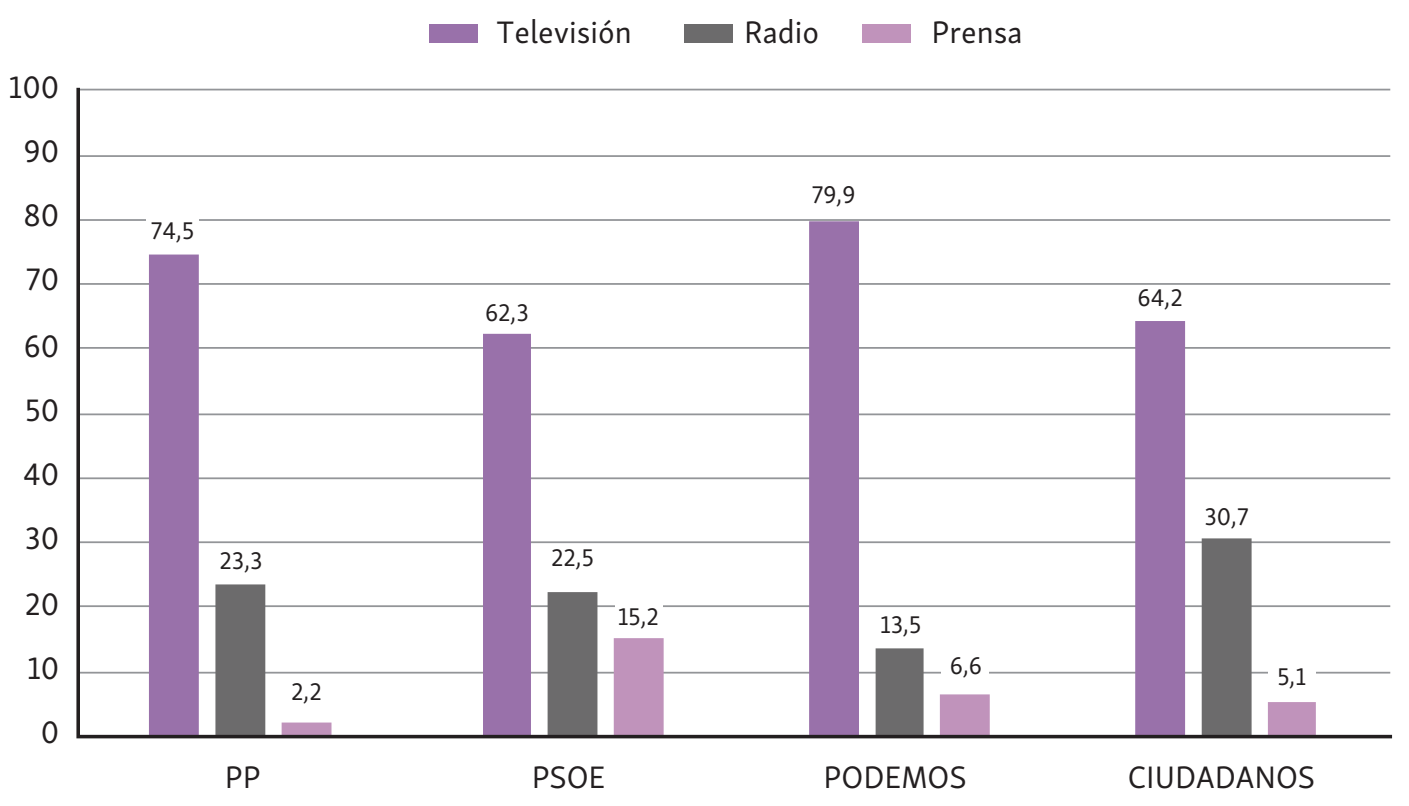

Figura 3. Presencia de medios durante los días de más actividad (\%)

Fuente: Elaboración propia.

Respecto de los programas televisivos que más protagonismo asumen en las cuentas de los partidos durante los días de mayor actividad, destacan tres espacios: el debate electoral del 13 de junio con los candidatos de los cuatro principales partidos, emitido por 17 canales, Al Rojo Vivo de La Sexta y Las Mañanas de Cuatro.

Sin tener en cuenta los tuits relacionados con el debate celebrado el 13 de junio y emitido en diferentes canales, Atresmedia (La Sexta y Antena 3) es el grupo que consigue una mayor atención por parte de los políticos. De los 1341 tuits que hacen referencia a intervenciones televisivas, $32 \%$ se dirige a estos canales. Por el contrario, las menciones al grupo Mediaset (Tele 5 y Cuatro) son menos abundantes, pues solo suman 15,7\%. Por su parte, Televisión Española (TVE) consigue una presencia de $13,9 \%$, gracias al debate a siete del 20 de junio, y a programas como Los Desayunos, La Mañana y La noche $24 \mathrm{H}$.

No obstante, si se atiende a la conducta específica de cada partido, se aprecian algunas diferencias destacables. Por ejemplo, el PP publica más mensajes relacionados con el programa estrella de la televisión pública (Los Desayunos de TVE) que con los referentes informativos de Atresmedia, Al Rojo Vivo y Espejo Público. PSOE, Podemos y Ciudadanos hacen lo contrario. Además, se observa que únicamente PP y Ciudadanos aluden a espacios del canal privado 13TV, del mismo modo que solo el PSOE y Podemos mencionan a Canal Sur, la televisión pública de Andalucía. 
Por último, cabe destacar la participación de Mariano Rajoy (PP) en programas que recurren a un tono más amable y personal en sus entrevistas, como El Hormiguero o Dos días y una noche, ambos de Antena 3. El resto de candidatos también intervinieron en estos espacios, pero lo hicieron antes del inicio oficial de la campaña electoral o en días que generaron poca actividad en Twitter. Esto explicaría que las referencias de PSOE, Podemos o Ciudadanos a dichos programas no figuren en esta investigación.

\section{CONCLUSIONES}

La importancia de esta investigación reside en la escasez de estudios específicos sobre la hibridación en las estrategias comunicativas de los actores políticos. Existen algunos trabajos sobre las aportaciones de la ciudadanía en redes sociales durante la emisión de debates electorales (Marcos-García et al., 2016), pero apenas se ha abordado la cuestión desde la perspectiva de los partidos políticos. Por ello, este artículo evalúa la relación entre viejos y nuevos medios que desarrollan las formaciones políticas en los medios sociales. Específicamente, se analiza esta relación híbrida durante la campaña de las elecciones generales de 2016 en España a partir de la actividad en Twitter de los cuatro partidos políticos más votados: PP, PSOE, Podemos y Ciudadanos.

Los resultados confirman que estos partidos utilizan Twitter como una extensión de sus herramientas tradicionales de comunicación, con el objetivo de promocionar sus intervenciones en medios convencionales, especialmente en televisión, para viralizar y amplificar el impacto de su discurso en campaña electoral, en consonancia con lo que han afirmado otros autores (Alonso-Muñoz et al., 2016; López-Meri et al., 2017).

En relación con la PI1 (¿Coinciden los picos de actividad de los partidos en Twitter con la celebración de eventos mediados por medios convencionales?), se aprecia que, en gran medida, los días de mayor actividad en Twitter, en términos de producción, suelen coincidir con eventos mediados. Dicho de otro modo, es frecuente que las jornadas que superan la media diaria de tuits se correspondan con días en los que los partidos registran más cantidad de apariciones en medios convencionales, del candidato o de otros miembros de la formación. Así ocurre en siete jornadas de la campaña electoral, en las que alguno de los partidos dedica, al menos, la mitad de sus tuits a contenidos mediáticos. En diversas jornadas, incluso se supera el $60 \%$. Además, el 13 de junio, día del debate de los cuatro candidatos, emitido por diversas televisiones, los contenidos mediáticos superan el 80\% en el perfil de Ciudadanos y $90 \%$ en la cuenta de Podemos. De hecho, la jornada del debate es 
uno de los picos de actividad comunes en los perfiles de los cuatro partidos. Estos hallazgos confirman la primera hipótesis planteada al inicio del artículo.

Por otra parte, respecto de la PI2 y el rol específico que juega cada tipo de medio, se demuestra que la televisión tiene más protagonismo que la radio y la prensa durante los días de mayor producción en Twitter. Este resultado confirma la segunda hipótesis. Todos los partidos incluyen más contenidos televisivos que radiofónicos. La prensa ocupa el último lugar. Por lo tanto, se legitima la función que cumple la televisión, que sigue copando la atención de los políticos como un valioso canal para dirigirse a la ciudadanía (López-Meri et al., 2017). En el entorno digital, los partidos siguen dando mucha importancia a la televisión. De hecho, se ayudan de las potencialidades de Twitter para captar más público y viralizar los contenidos mediáticos de los que son protagonistas, según se ha visto en investigaciones anteriores (Chadwick, 2017; Casero-Ripollés et al., 2016).

El dominio de la televisión puede explicarse, en parte, por la relevancia que adquieren los dos debates televisados durante la campaña, especialmente el ya mencionado con los cuatro candidatos de los principales partidos. No obstante, también tienen un peso destacado los magacines de actualidad política y las tertulias matinales, sobre todo Al Rojo Vivo (Atresmedia) y Las Mañanas de Cuatro (Mediaset).

Por último, respecto de la PI3, no existen diferencias sustanciales entre los partidos clásicos y las fuerzas emergentes. Tanto PP y PSOE como Podemos y Ciudadanos dan prioridad a sus intervenciones en televisión, por lo que queda refutada la tercera hipótesis. Únicamente Ciudadanos concede algo más de espacio a contenidos radiofónicos, aunque dedica el doble de tuits a programas de televisión. Sin embargo, sí se aprecia que los partidos de tendencia liberal, PP y Ciudadanos, destinan más mensajes a promocionar sus intervenciones en medios que sus actos de campaña. Las fuerzas de izquierdas, PSOE y Podemos, hacen lo contrario y prestan el doble de atención a sus mítines que a sus apariciones en medios.

En conclusión, estos resultados refuerzan la idea de que Twitter funciona como un espacio híbrido donde coexisten en equilibrio las lógicas de transmisión y recepción de los medios convencionales y las nuevas lógicas de circulación y negociación que ofrece el contexto digital (Chadwick, 2017; Graham et al., 2013). Lejos de sustituir a los medios tradicionales, Twitter los complementa y enriquece las estrategias comunicativas de los partidos políticos, en un proceso de integración en el que nuevos y viejos medios conviven, interaccionan y cooperan de forma dinámica (Chadwick, 2011; Casero-Ripollés et al., 2016).

No obstante, aunque estas conclusiones son extrapolables a otros contextos, es necesario investigar más en esta línea, por ejemplo, analizando la hibridación de la comunicación política en periodos diferentes a las campañas electorales. 


\section{REFERENCIAS}

Alonso, M. A. \& Adell, A. (2011). Marketing Político 2.0: Lo que todo candidato necesita saber para ganar las elecciones (Political Marketing 2.0: What Every Candidate Needs to Know to Win the Election). Barcelona, Spain: Gestión 2000.

Alonso-Muñoz, L. \& Casero-Ripollés, A. (2018). Political agenda on Twitter during the 2016 Spanish elections: issues, strategies, and users' responses. Communication $\mathcal{E}$ society, 31(3), 7-23. https://doi.org/10.15581/003.31.3.7-23

Alonso-Muñoz, L., Marcos-García, S., \& Casero-Ripollés, A. (2017). Political leaders in (inter) action. Twitter as a strategic communication tool in electoral campaigns. Trípodos, (39), 71-90. Retrieved from http://www.tripodos.com/index.php/Facultat_Comunicacio_ Blanquerna/article/view/381

Alonso-Muñoz, L., Miquel Segarra, S., \& Casero-Ripollés, A. (2016). Un potencial comunicativo desaprovechado. Twitter como mecanismo generador de diálogo en campaña electoral (An untapped communicative potential. Twitter as a dialogue generator mechanism in electoral campaign). Obra digital: revista de comunicación, (11), 39-59. https://doi.org/10.25029/od.2016.100.11

Anstead, N. \& O'Loughlin, B. (2011). The emerging viewertariat and BBC question time: Television debate and real-time commenting online. The International Journal of Press/Politics, 16(4), 440-462. https://doi.org/10.1177/1940161211415519

Bentivegna, S. (2015). A colpi di tweet: la politica in prima persona (With tweets: politics in the first person). Bologna, Italy: Il Mulino.

Broersma, M. \& Graham, T. (2013). Twitter as a news source: How Dutch and British newspapers used tweets in their news coverage, 2007-2011. Journalism Practice, 7(4), 446-464. https://doi.org/10.1080/17512786.2013.802481

Casero-Ripollés, A. (2008). Modelos de relación entre periodistas y políticos: La perspectiva de la negociación constante (Models of relationship between journalists and politicians: The perspective of the constant negotiation). Estudios sobre el mensaje periodistico, 14, 111-129. Retrieved from https://revistas.ucm.es/index.php/ESMP/article/view/ESMP0808110111A

Casero-Ripollés, A. (2018). Research on political information and social media: Key points and challenges for the future. El profesional de la información, 27(5). https://doi.org/10.3145/ epi.2018.sep.01

Casero-Ripollés, A. (2020). Influencia de los medios de comunicación en la conversación política en Twitter (Influence of media on the political conversation on Twitter: Activity, popularity, and authority in the digital debate in Spain). Revista ICONO14 Revista cientifica de Comunicación y Tecnologías emergentes, 18(1), 33-57. https://doi.org/10.7195/ri14.v18i1.1527

Casero-Ripollés, A., Feenstra, R. A., \& Tormey, S. (2016). Old and new media logics in an electoral campaign: The case of Podemos and the two-way street mediatization of politics. The International Journal of Press/Politics, 21(3), 378-397. https://doi.org/10.1177/1940161216645340

Ceccarini, L. (2015). La cittadinanza in rete (Citizenship on the net). Bologna, Italy: Il Mulino.

Cha, M., Haddadi, H., Benevenuto, F., \& Gummadi, K. P. (2010, May). Measuring user influence in twitter: The million follower fallacy. In Proceedings of the Fourth International AAAI Conference on Weblogs and Social Media (ICWSM 2010) (pp. 10-17). Menlo Park, CA: AAAI Press. 
Chadwick, A. (2017). The hybrid media system: Politics and power. Oxford, UK: Oxford University Press.

Chadwick, A. (2011). The political information cycle in a hybrid news system: The British prime minister and the "Bullygate" affair. The International Journal of Press/Politics, 16(1), 3-29. https://doi.org/10.1177/1940161210384730

Criado, J. I., Martínez-Fuentes, G., \& Silván, A. (2013). Twitter en campaña: las elecciones municipales españolas de 2011 (Twitter in campaign: the 2011 Spanish municipal elections). RIPS. Revista de Investigaciones Políticas y Sociológicas, 12(1), 93-113. Retrieved from https://revistas.usc.gal/index.php/rips/article/view/1307

Doménech, H. (2018). Las claves para la gestión de la imagen en política (The keys to image management in politics). adComunica, (16), 261-263. https://doi.org/10.6035/533

Freelon, D. \& Karpf, D. (2015). Of big birds and bayonets: Hybrid Twitter interactivity in the 2012 presidential debates. Information, Communication \& Society, 18(4), 390-406. https://doi.org/10.1080/1369118X.2014.952659

Gainous, J. \& Wagner, K. M. (2014). Tweeting to power: The social media revolution in American politics. Oxford, UK: Oxford University Press.

Graham, T., Broersma, M., Hazelhoff, K., \& Van'T Haar, G. (2013). Between broadcasting political messages and interacting with voters: The use of Twitter during the $2010 \mathrm{UK}$ general election campaign. Information, Communication \& Society, 16(5), 692-716. https://doi.org/10.1080/1369118X.2013.785581

Hanna, A., Wells, C., Maurer, P., Friedland, L., Shah, D., \& Matthes, J. (2013). Partisan alignments and political polarization online: A computational approach to understanding the French and US presidential elections. In I. Weber, A. M. Popescu, \& M. Pennacchiotti (Eds.), Proceedings of the 2nd workshop politics, elections and data (pp. 15-21). New York, NY: ACM.

Holtz-Bacha, C. (2013). Web 2.0: nuevos desafíos en comunicación política (Web 2.0: new challenges in political communication). Diálogo político, 30(1), 11-27.

Hosch-Dayican, B. (2014). Online political activities as emerging forms of political participation: How do they fit in the conceptual map?Acta politica, 49, 342-346. https://doi.org/10.1057/ap.2014.7

Iannelli, L. (2016). Hybrid Politics: Media and Participation. London, UK: SAGE.

Jackson, N. \& Lilleker, D. (2011). Microblogging, constituency service and impression management: UK MPs and the use of Twitter. The journal of legislative studies, 17(1), 86-105. https://doi.org/10.1080/13572334.2011.545181

Jenkins, H. (2006). Convergence culture: Where old and new media collide. New York, NY: NYU Press.

Jewitt, R. (2009). The trouble with twittering: Integrating social media into mainstream news. International Journal of Media and Cultural Politics, 5(3), 233-240.

Jungherr A. (2014). Twitter in politics: a comprehensive literature review. Retrieved from https://papers.ssrn.com/sol3/papers.cfm?abstract_id=2865150

Kalsnes, B., Krumsvik, A.H., \& Storsul, T. (2014). Social media as a political backchannel: Twitter use during televised election debates in Norway. Aslib Journal of Information Management, 66(3), 313-328. https://doi.org/10.1108/AJIM-09-2013-0093 
Karlsen, R. \& Enjolras, B. (2016). Styles of social media campaigning and influence in a hybrid political communication system: Linking candidate survey data with Twitter data. The International Journal of Press/Politics, 21(3), 338-357. https://doi.org/10.1177/1940161216645335

Kim, Y. (2011). The contribution of social network sites to exposure to political difference: The relationships among SNSs, online political messaging, and exposure to cross-cutting perspectives. Computersin Human Behavior, 27(2), 971-977. https://doi.org/10.1016/j.chb.2010.12.001

Kruikemeier, S. (2014). How political candidates use Twitter and the impact on votes. Computers in human behavior, 34, 131-139. https://doi.org/10.1016/j.chb.2014.01.025

Larsson, A. O. (2016). Online, all the time? A quantitative assessment of the permanent campaign on Facebook. New media \& society, 18(2), 274-292. https://doi.org/10.1177/1461444814538798

Larsson, A. O. (2017). The news user on social media: A comparative study of interacting with media organizations on Facebook and Instagram. Journalism studies, 19(15), 2225-2242. https://doi.org/10.1080/1461670X.2017.1332957

Lilleker, D. G., Tenscher, J., \& Štětka, V. (2015). Towards hypermedia campaigning? Perceptions of new media's importance for campaigning by party strategists in comparative perspective. Information, Communication \& Society, 18(7), 747-765. https://doi.org/10.1080/1369118X.2014.993679

López-Meri, A. \& Casero-Ripollés, A. (2017). Las estrategias de los periodistas para la construcción de marca personal en Twitter: posicionamiento, curación de contenidos, personalización y especialización (Journalists' strategies to build personal brand on Twitter: positioning, content curation, personalization and specialisation). Revista Mediterránea de Comunicación, 8(1), 59-73. https://doi.org/10.14198/MEDCOM2017.8.1.5

López-Meri, A., Marcos-García, S., \& Casero-Ripollés, A. (2017). What do politicians do on Twitter? Functions and communication strategies in the Spanish electoral campaign of 2016. El profesional de la información, 26(5), 795-804. Retrieved from http://www.elprofesionaldelainformacion.com/contenidos/2017/sep/02.pdf

Marchetti, R. \& Ceccobelli, D. (2016). Twitter and television in a hybrid media system: the 2013 Italian election campaign. Journalism Practice, 10(5), 626-644. https://doi.org/10.1080/17512786.2015.1040051

Marcos-García, S. \& Alonso-Muñoz, L. (2019). ¿Elecciones del cambio? Gestión estratégica de Twitter en los comicios municipales de Madrid 2015 (Elections of change? Strategic management of Twitter in the municipal elections of Madrid 2015). In J. Sierra Sánchez \& S. Liberal Ormaechea (Coords.), Uso y aplicación de las redes sociales en el mundo audiovisual $y$ publicitario (Use and application of social networks in the audiovisual and advertising world) (pp. 107-118). Madrid, Spain: McGraw-Hill.

Marcos-García, S., Alonso-Muñoz, L., \& Casero-Ripollés, A. (2017). Usos ciudadanos de Twitter en eventos políticos relevantes. La \#SesiónDeInvestidura de Pedro Sánchez (Citizen uses of Twitter in important political events. The \#SesiónDeInvestidura of Pedro Sánchez). Comunicación y Hombre, (13), 25-49. Retrieved from https://comunicacionyhombre.com/article/usos-de-twitter-en-eventos-politicos/

Mascaro, C. \& Goggins, S. P. (2012). Twitter as virtual town square: Citizen engagement during a nationally televised Republican primary debate. In APSA 2012 Annual Meeting Paper. Retrieved from https://ssrn.com/abstract=2108682 
Murthy, D. (2013). Twitter: Social communication in the Twitter age. Hoboken, NJ: John Wiley \& Sons.

Norquay, G. (2008). Organizing without an organization: The Obama networking revolution. Policy Options, 29(9), 58-61.

Papacharissi, Z. \& de Fatima Oliveira, M. (2011, September). The rhythms of news storytelling on Twitter: Coverage of the January 25th Egyptian uprising on Twitter. In World Association for Public Opinion Research Conference (Vol. 312, p. 3188).

Parmelee, J. H. \& Bichard, S. L. (2011). Politics and the Twitter revolution: How tweets influence the relationship between political leaders and the public. United Kingdom: Lexington Books.

Pedersen, S., Baxter, G., Burnett, S., Goker, A., Corney, D., \& Martin, C. (2020). Backchannel chat: peaks and troughs in a Twitter response to three televised debates during the Scottish Independence Referendum campaign 2014. Aberdeen Business School Working Paper Series, 7(2). Retrieved from https://openair.rgu.ac.uk/handle/10059/1086

Pérez-Curiel, C. \& García-Gordillo, M. (2020). Del debate electoral en TV al ciberdebate en Twitter. Encuadres de influencia en las elecciones generales en España (28A) (From TV debate to cyber debate on Twitter: frames of influence in the general elections in Spain (28A)). El Profesional de la información, 29(4), e290405. https://doi.org/10.3145/epi.2020.jul.05

Piscitelli, A. (2011). Prólogo: Twitter, la revolución y los enfoques ni-ni (Prologue: Twitter, the revolution and the ni-ni approaches). In J. L. Orihuela, Mundo Twitter (Twitter World) (pp. 15-20). Madrid, Spain: Alienta Editorial.

Rega, R. (2016). Twitter as a New Engagement Opportunity. Analysis of the Questions and Answers between the Italian Prime Minister and Citizens. Trípodos, (39), 91-107. Retrieved fromhttp://www.tripodos.com/index.php/Facultat_Comunicacio_Blanquerna/article/view/382

Rodríguez, R. \& Ureña, D. (2011). Diez razones para el uso de Twitter como herramienta en la comunicación política y electoral (Ten reasons to use Twitter as a tool for political and electoral communication). Comunicación y pluralismo, 10, 89-115. Retrieved from https://summa.upsa.es/viewer.vm?id=30573\&view=main\&lang=es

Rossi, L. \& Magnani, M. (2012, May). Conversation practices and network structure in Twitter. In Sixth International AAAI Conference on Weblogs and Social Media (pp. 563-566).

Rúas, X. \& Casero-Ripollés, A. (2018). Comunicación política en la época de las redes sociales: lo viejo y lo nuevo, y más allá (Political communication in the age of social networks: the old and the new, and beyond). adComunica, (16), 21-24. Retrieved from http://www.erevistes.uji.es/index.php/adcomunica/article/view/4984

Shamma, D. A., Kennedy, L., \& Churchill, E. F. (2009, October). Tweet the debates: understanding community annotation of uncollected sources. In Proceedings of the first SIGMM workshop on Social media (pp. 3-10). ACM. https://doi.org/10.1145/1631144.1631148

Strömbäck, J. (2008). Four phases of mediatization: An analysis of the mediatization of politics. The International Journal of Press/Politics, 13(3), 228-246. https://doi.org/10.1177/1940161208319097

Stromer-Galley, J. (2014). Presidential campaigning in the Internet age. Oxford, UK: Oxford University Press. 
Suau-Gomila, G. \& Pont-Sorribes, C. (2019). Microblogging electoral: los usos de Twitter de Podemos y Ciudadanos y sus líderes Pablo Iglesias y Albert Rivera en las elecciones generales españolas de 2016 (Electoral microblogging: the uses of Twitter by Podemos and Ciudadanos and its leaders Pablo Iglesias and Albert Rivera in the Spanish general elections of 2016). Estudios sobre el mensaje periodístico, 25(2), 1121. https://doi.org/10.5209/esmp.64829

Trilling, D. (2015). Two different debates? Investigating the relationship between a political debate on TV and simultaneous comments on Twitter. Social Science Computer Review, 33(3), 259-276. https://doi.org/10.1177/0894439314537886

Vergeer, M., Hermans, L., \& Sams, S. (2013). Online social networks and micro-blogging in political campaigning: The exploration of a new campaign tool and a new campaign style. Party Politics, 19(3), 477-501. https://doi.org/10.1177/1354068811407580

Wohn, D. Y. \& Na, E. K. (2011). Tweeting about TV: Sharing television viewing experiences via social media message streams. First Monday, 16(3). https://doi.org/10.5210/fm.v16i3.3368

\section{SOBRE LAS AUTORAS}

SILVIA MARCOS GARCíA, doctora en Ciencias de la Comunicación por la Universitat Jaume I (UJI), donde actualmente ejerce como profesora ayudante doctora. Es graduada en Periodismo con premio extraordinario final de carrera y máster en Nuevas Tendencias y Procesos de Innovación en Comunicación por la UJI. Sus líneas de investigación se centran en el estudio del periodismo, las redes sociales y la comunicación política desde las perspectivas de los periodistas, actores políticos y ciudadanía.

iD http://orcid.org/0000-0003-1682-1009

LAURA ALONSO-MUÑOz, doctora en Ciencias de la Comunicación por la Universitat Jaume I (UJI), actualmente es profesora ayudante doctora en la misma universidad. Asimismo, posee un máster en Nuevas Tendencias, es graduada en Periodismo por la UJI y licenciada en Ciencias Políticas y de la Administración por la Universitat Pompeu Fabra de Barcelona. Sus investigaciones abordan el uso de las redes sociales por parte de actores políticos, ciudadanos y medios de comunicación, y el fenómeno populista en el entorno digital.

iD http://orcid.org/0000-0001-8894-1064

AMPARO LóPEZ-MERI, doctora en Ciencias de la Comunicación por la Universitat Jaume I (España) y profesora ayudante en la misma universidad. Previamente, obtuvo la licenciatura en Periodismo por la Universidad CEU San Pablo y un máster oficial en Nuevas Tendencias e Innovación en Comunicación. Ha sido galardonada con el Premio Lorenzo Gomis de la Sociedad Española de Periodismo. Sus investigaciones analizan la reformulación del periodismo y la comunicación política en el entorno digital, así como la ética de los medios.

iD http://orcid.org/0000-0003-3408-2190 